\title{
Acute biliary pancreatitis has better outcomes but increased resource utilization compared to acute alcohol-induced pancreatitis: insights from a nationwide study
}

\author{
Sachit Sharma a, Christian Nehmea, Muhammad Aziz ${ }^{a}$, Simcha Weissman ${ }^{b}$, Ahmad Khanc, \\ Ashu Acharyaa, Ishaan Vohrad , Sami Ghazaleha ${ }^{a}$, Ali Nawrase, Douglas G. Adlerf \\ University of Toledo Medical Center, Toledo, OH; Hackensack Meridian Health Palisades Medical Center, North \\ Bergen, NJ; West Virginia University Health Sciences Center Charleston Division, WV; John H. Stroger Jr, Hospital of \\ Cook Country, Chicago, IL; University of Utah, Salt Lake City, UT, USA
}

\section{Abstract}

Background The differences in outcomes between acute biliary pancreatitis (ABP) and acute alcohol-induced pancreatitis (AAP) have not been well studied. We sought to examine the differences between ABP and AAP as regards to in-hospital outcomes and resource utilization, using a large nationwide database.

Methods We queried the National Inpatient Sample databases 2016 and 2017 using the International Classification of Diseases, 10th revision, Clinical Modification (ICD-10-CM) coding system to identify the patients with a primary diagnosis of AAP and ABP. The primary outcome was all-cause in-hospital mortality. Secondary outcomes were hospital length of stay (LOS), hospitalization charge/cost, shock, acute kidney injury (AKI), intensive care unit (ICU) admission, and home discharge. Analysis was performed with STATA software.

Results There was no significant difference in mortality between patients with AAP and ABP $(0.42 \%$ vs. $0.82 \%$, adjusted odds ratio [aOR] $0.95,95 \%$ confidence interval $[\mathrm{CI}] 0.69-1.31 ; \mathrm{P}=0.79)$. Patients with ABP had a significantly longer LOS ( +0.48 days, $\mathrm{P}<0.001)$. Patients with ABP had significantly higher adjusted mean hospitalization charges $(\$+19,958, \mathrm{P}<0.001)$ and costs $(\$+4,848, \mathrm{P}<0.001)$. Patients with ABP had a significantly lower likelihood of shock (aOR 0.75, 95\%CI 0.59-0.95; $\mathrm{P}=0.02$ ), AKI (aOR 0.76, 95\%CI 0.71-0.82; P<0.001) or ICU admission (aOR 0.74, 95\%CI 0.62-0.88; P=0.001). They were more likely to be discharged home (aOR 1.26, 95\%CI 1.18-1.34; $\mathrm{P}<0.001$ ).

Conclusion Although there was no difference in all-cause mortality, patients with ABP had better hospitalization outcomes but greater resource utilization.

Keywords Acute biliary pancreatitis, acute alcohol-induced pancreatitis, mortality, outcomes, resource utilization

Ann Gastroenterol 2021; 34 (1): 1-10

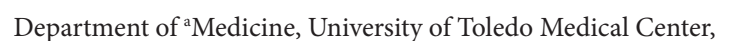
Toledo, OH (Sachit Sharma, Christian Nehme, Muhammad Aziz, Ashu Acharya, Sami Ghazaleh); ${ }^{b}$ Medicine, Hackensack Meridian Health Palisades Medical Center, North Bergen, NJ (Simcha Weissman); ${ }^{c}$ Medicine, West Virginia University Health Sciences Center Charleston Division, Charleston, WV (Ahmad Khan); ${ }^{\mathrm{d} M e d i c i n e, ~ J o h n ~ H . ~}$ Stroger Jr, Hospital of Cook County, Chicago, IL (Ishaan Vohra); ${ }^{\mathrm{e}} \mathrm{Gastroenterology,} \mathrm{The} \mathrm{University} \mathrm{of} \mathrm{Toledo} \mathrm{Medical} \mathrm{Center,} \mathrm{Toledo,}$ $\mathrm{OH}$ (Ali Nawras); f Gastroenterology, University of Utah, Salt Lake City, UT (Douglas G. Adler), USA

\section{Conflict of Interest: None}

Correspondence to: Douglas G. Adler, MD, University of Utah, Salt Lake City, Utah, United States, e-mail: Douglas.Adler@hsc.utah.edu

Received 1 June 2020; accepted 1 September 2020; published online 7 December 2020

DOI: https://doi.org/10.20524/aog.2020.0559

\section{Introduction}

Acute pancreatitis (AP) is an acute inflammatory process involving the pancreas with considerable morbidity and mortality [1]. Global incidence is estimated to be about 34 cases per 100,000 population per year, with no significant sex-related differences [2]. The prevalence of AP has more than doubled in recent years, and AP is considered one of the most common gastrointestinal cause for hospitalization in the United States (US) [3]. AP poses a significant economic burden on the US healthcare system, with more than $\$ 10$ billion in estimated costs annually, and this trend is increasing $[4,5]$.

The most common cause of AP is biliary stone disease (choledocholithiasis) followed by alcohol-induced pancreatitis. Together, they account for almost $70-80 \%$ 
of all cases of AP [6-8]. Other causes of AP include postendoscopic retrograde cholangiopancreatography pancreatitis, medications, hypertriglyceridemia, hypercalcemia, trauma, infections, autoimmune, genetic, and idiopathic [7]. When comparing outcome differences between 2 different etiologies of AP, acute alcohol-induced pancreatitis (AAP) and acute biliary pancreatitis (ABP), some studies have reported a more severe outcome among patients with ABP $[9,10]$, while other studies have reported a more severe course and higher mortality in patients with AAP $[11,12]$. Other studies have reported no difference in mortality between the 2 groups $[8,13]$.

We performed a retrospective analysis of the Nationwide Inpatient Sample (NIS) to compare the clinical course and outcomes between patients with AAP and ABP.

\section{Materials and methods}

\section{Data source}

We queried the Healthcare Cost and Utilization Project (HCUP) NIS database for the years 2016 and 2017. The NIS is a database of inpatient hospital stays in the United States (excluding rehabilitation and long-term acute care hospitals) derived from billing data submitted (by hospitals) to statewide organizations based off discharge abstracts. The NIS 2016 database contains data from 7.1 million hospital stays in 4575 hospitals in 47 US states, while the NIS 2017 database contains data from 7.1 million hospital stays in 4584 hospitals in 48 US states [14]. It contains de-identified clinical and nonclinical elements at both the patient and hospital level using the International Classification of Diseases, 10th revision, Clinical Modification (ICD-10-CM) coding system. The current study was performed using the ICD-10-CM codes, which are more specific than the previous iteration, in place prior to the years examined [15]. NIS 2017 has an increased number of secondary diagnoses (29-39) and inpatient procedures (15-25), which allowed us to generate a more comprehensive list of patients and procedures than the previous NIS databases.

\section{Study population}

We used the ICD-10-CM codes to identify the following: (a) patients admitted with a primary diagnosis of AP; and (b) patients admitted with a primary diagnosis of AAP or ABP. Patients were excluded if they were $<18$ years old or if they had elective admission. The ICD-10-CM diagnostic and procedural codes used in this study are presented in the Supplementary Table 1. Inclusion and exclusion criteria are shown in the flow diagram (Fig. 1). This study was deemed exempt from institutional review board approval at the University of Toledo, as it was performed using de-identified and publicly available data.

\section{Study variables}

Patient demographics collected included: age, sex, race (Caucasian, Black, Hispanic, Asian, Pacific Islander, Native American, and other), median household income (based on patient's zip code), primary expected payer (Medicare, Medicaid, private insurance, and uninsured), hospital size (small, medium, and large) in terms of number of beds, hospital teaching status, hospital region (Northeast, Midwest, South, and West), and urban location. The burden of comorbidities was assessed using the Charlson comorbidity index. Mortality rate, patient discharge, hospital length of stay (LOS), total charges (the amount billed by the hospital for the rendered services), as well as the (actual) cost of care were all obtained directly from the NIS. Total hospitalization charge is the amount billed to the patient and is directly available in the NIS, but is different from hospitalization cost, which is the actual cost incurred by the hospital in treating the patient $[16,17]$. A cost-tocharge ratio is available from HCUP and can be used to calculate the hospitalization cost [18].

\section{Study outcomes}

The primary outcome was in-hospital all-cause mortality. Secondary outcomes were: (a) mean LOS; (b) mean hospitalization charges and costs; (c) shock; (d) sepsis; (e) acute kidney injury (AKI); (f) intensive care unit (ICU) admission; (g) likelihood of pancreatic drainage; and (h) likelihood of discharge to home. All these outcomes were defined using standard ICD-10-CM diagnostic and procedural codes, as shown in the Supplementary Table 1.

\section{Statistical analysis}

Statistical analysis was performed using STATA, version 16.0 (StataCorp., College Station, Texas, US). This software facilitates analysis to produce nationally representative unbiased results, variance estimates and P-values. Weighting of patient-level observations was implemented. NIS contains a $20 \%$ sample of all hospitals within each stratum. During analysis, this sample was weighted (weight $=$ total number of discharges from all acute care hospitals in the US divided by the number of discharges included in the $20 \%$ sample) to obtain nationally representative data [19]. Multivariate regression analysis was used to adjust for potential confounders. Univariate analysis was initially performed to calculate the unadjusted odds ratio $(\mathrm{OR})$ and determine confounders significantly associated with the outcomes. Significant association was determined as a cutoff P-value of 0.2. A multivariate regression model was then constructed, including all confounders found to be significant by univariate analysis-age, sex, race, comorbidity score, insurance status, hospital region, hospital location, income quartile, congestive 
Table 1 Characteristics of patients admitted to hospital with acute pancreatitis

\begin{tabular}{|c|c|}
\hline Variable & $\mathrm{n}(\%)$ \\
\hline Total study population & 553,480 \\
\hline Female sex & $258,917(46.78)$ \\
\hline Mean age in years & 51.8 \\
\hline \multicolumn{2}{|l|}{ Insurance provider } \\
\hline Medicare & $176,670(31.92)$ \\
\hline Medicaid & $141,525(25.57)$ \\
\hline Private & $183,534(33.16)$ \\
\hline Uninsured & $51,805(9.36)$ \\
\hline \multicolumn{2}{|l|}{ Charlson comorbidity index } \\
\hline 0 & $225,487(40.74)$ \\
\hline 1 & $165,213(29.85)$ \\
\hline 2 & $76,435(13.81)$ \\
\hline 3 or more & $86,343(15.6)$ \\
\hline \multicolumn{2}{|c|}{ Median income in patient zip code } \\
\hline$\$ 1-\$ 38,999$ & $181,596(32.81)$ \\
\hline$\$ 39,000-\$ 47,999$ & $147,170(26.59)$ \\
\hline$\$ 48,000-\$ 62,999$ & $128,905(23.29)$ \\
\hline$\$ 63,000$ & $95,752(17.3)$ \\
\hline \multicolumn{2}{|l|}{ Hospital region } \\
\hline Northwest & $95,475(17.25)$ \\
\hline Midwest & $123,647(22.34)$ \\
\hline South & $225,155(40.68)$ \\
\hline West & $109,201(19.73)$ \\
\hline \multicolumn{2}{|l|}{ Hospital location } \\
\hline Rural & $63,318(11.44)$ \\
\hline Urban & $490,162(88.56)$ \\
\hline \multicolumn{2}{|l|}{ Hospital size } \\
\hline Small & $127,245(22.99)$ \\
\hline Medium & $171,247(30.94)$ \\
\hline Large & $254,988(46.07)$ \\
\hline Teaching hospital & $334,966(60.52)$ \\
\hline \multicolumn{2}{|l|}{ Race } \\
\hline White & $355,500(64.23)$ \\
\hline Black & $95,254(17.21)$ \\
\hline Hispanic & $70,790(12.79)$ \\
\hline Asian or Pacific Islander & $11,402(2.06)$ \\
\hline Native American & $4,649(0.84)$ \\
\hline Other & $15,830(2.86)$ \\
\hline \multicolumn{2}{|l|}{ Comorbidities } \\
\hline CHF & $30,884(5.58)$ \\
\hline Cirrhosis & $19,372(3.5)$ \\
\hline
\end{tabular}

Table 1 (Continued)

\begin{tabular}{lc}
\hline Variable & $\mathrm{n}(\%)$ \\
\hline Type 1 diabetes & $6,365(1.15)$ \\
Type 2 diabetes & $139,864(25.27)$ \\
CKD & $50,034(9.04)$ \\
ESRD & $11,900(2.15)$ \\
Obese & $94,036(16.99)$ \\
Smoker & $265,781(48.02)$ \\
\hline
\end{tabular}

CHF, congestive heart failure; $C K D$, chronic kidney disease; ESRD, end-stage renal disease

heart failure (CHF), cirrhosis, chronic kidney disease (CKD), end-stage renal disease (ESRD), diabetes mellitus (DM), obesity - to calculate the adjusted OR (aOR). Logistic regression was used for binary outcomes and linear regression was used for continuous outcomes. Proportions were compared using the chi-square test and continuous variables were compared using the Student's $t$-test. A P-value $<0.05$ was considered statistically significant for all outcomes.

\section{Results}

\section{Patient characteristics}

A total of 585,579 adult patient admissions were identified with a diagnosis of AP during hospitalization in 2016 and 2017. Patients under the age of 18 years $(10,350)$ and with elective admission $(21,749)$ were excluded from the study, leaving 553,480 adult patients admitted with AP. Mean age was 51.8 years, and most of the patients were male (53.22\%). Private insurance was the patients' primary payer $(33.16 \%)$, followed by Medicare (31.92\%). The majority of patients were white $(64.23 \%)$. Patients were predominantly admitted to teaching hospitals (60.52\%). Just over one quarter $(25.3 \%)$ of the patients had AAP and $17.44 \%$ patients had ABP (Fig. 2). The complete patient and hospital characteristics are presented in Tables 1 and 2, and the outcomes are presented in Table 3.

Patients with ABP were more likely to be female compared to patients with $\operatorname{AAP}(59.4 \%$ vs. $30.73 \%, \mathrm{P}<0.001)$, and were older (57.03 vs. 45.31 years, $\mathrm{P}<0.001$ ). Patients with $\mathrm{ABP}$ had a higher prevalence of $\mathrm{CHF}(7.76 \%$ vs. $2.75 \%, \mathrm{P}<0.001)$, type $2 \mathrm{DM}(22.37 \%$ vs. $13.11 \%, \mathrm{P}<0.001)$, CKD (10.19\% vs. $3.25 \%$, $\mathrm{P}<0.001)$, ESRD (1.61\% vs. $0.34 \%, \mathrm{P}<0.001)$, and obesity (26.26\% vs. $8.24 \%, \mathrm{P}<0.001)$. Patients with AAP had a higher prevalence of liver cirrhosis $(5.55 \%$ vs. $2.5 \%, \mathrm{P}<0.001)$ and smoking $(66.17 \%$ vs. $30.75 \%, \mathrm{P}<0.001)$. Patients with AAP had a higher prevalence of pancreatic necrosis $(2.62 \%$ vs. $1.97 \%)$, but this difference was not significant on multivariate analysis (aOR 0.89, 95\% confidence interval [CI] 0.76-1.05; $\mathrm{P}=0.19$ ). 


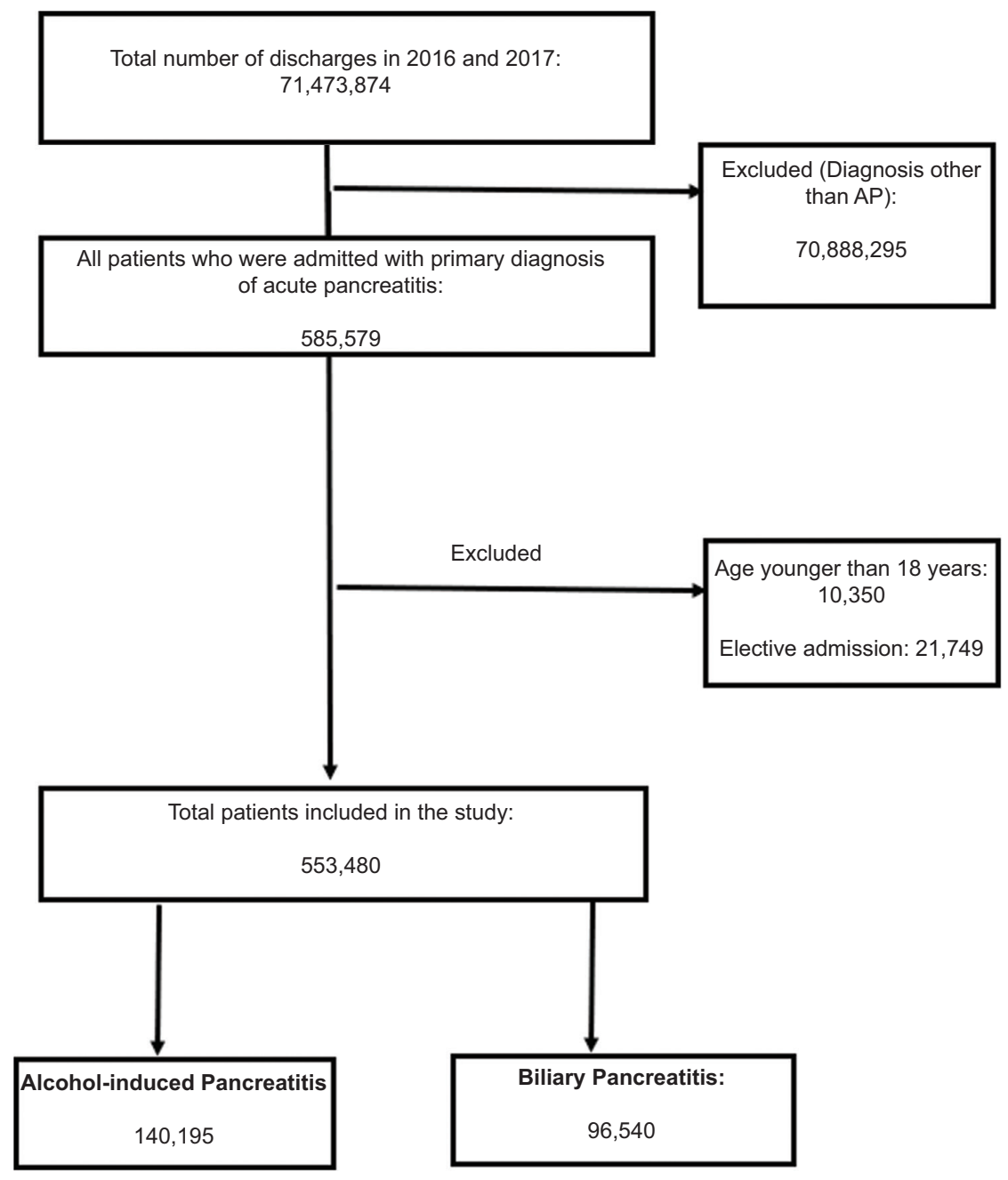

Figure 1 Patient selection flow diagram $A P$, acute pancreatitis

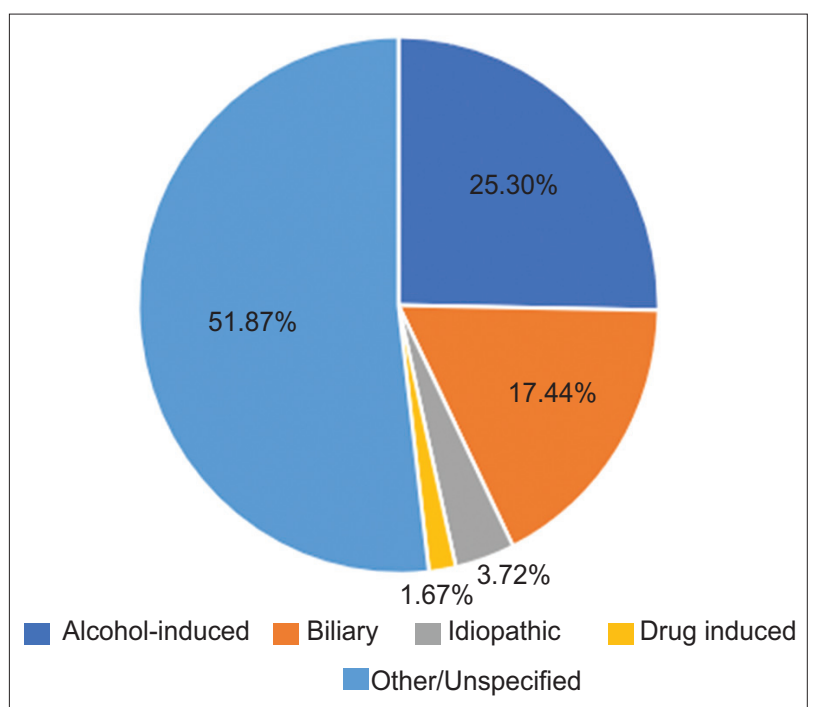

Figure 2 Etiology of acute pancreatitis in our National Inpatient Sample study

\section{All-cause in-hospital mortality}

The total all-cause in-hospital mortality in patients with AP was $0.59 \%$ (3285 of 553,480 patients). The total mortality was $0.42 \%$ (595 of 140,195 patients) among patients with AAP and $0.82 \%$ (800 of 96,540 patients) among patients with ABP. On multivariate analysis, there was no significant difference in all cause in-hospital mortality between patients with AAP and those with ABP (aOR 0.95, 95\%CI 0.69-1.31; $\mathrm{P}=0.79$ ).

\section{LOS}

The mean LOS among patients admitted with AP was 4.3 days. Mean LOS was 4.38 days (95\%CI 4.32-4.44) in patients with AAP and 5.1 days (95\%CI 5.01-5.18) in patients with ABP. After adjusting for confounders using multivariate regression analysis, patients with ABP had a significantly longer mean LOS compared to patients with AAP ( +0.48 days, $\mathrm{P}<0.001)$. 
Table 2 Characteristics of patients admitted with acute pancreatitis in hospital: acute biliary pancreatitis (ABP) versus acute alcohol-induced pancreatitis (AAP)

\begin{tabular}{|c|c|c|c|}
\hline \multirow[t]{2}{*}{ Variable } & \multicolumn{3}{|c|}{ n (\%) } \\
\hline & AAP $140,195(25.3 \%)$ & АВР 96,540 (17.44\%) & P-value \\
\hline Female sex & 30.73 & 59.4 & $<0.001$ \\
\hline Mean age in years & 45.31 & 57.03 & $<0.001$ \\
\hline Insurance provider & & & $<0.001$ \\
\hline Medicare & 14.43 & 40.82 & \\
\hline Medicaid & 37.99 & 18.17 & \\
\hline Private & 31.86 & 34.25 & \\
\hline Uninsured & 15.72 & 6.76 & \\
\hline Charlson comorbidity index & & & $<0.001$ \\
\hline 0 & 44.27 & 48.28 & \\
\hline 1 & 35.26 & 24.59 & \\
\hline 2 & 11.53 & 11.82 & \\
\hline 3 or more & 8.94 & 15.31 & \\
\hline Median income in patient zip code & & & $<0.001$ \\
\hline$\$ 1-\$ 38,999$ & 33.81 & 29.01 & \\
\hline$\$ 39,000-\$ 47,999$ & 25.43 & 26.63 & \\
\hline$\$ 48,000-\$ 62,999$ & 23.56 & 24.41 & \\
\hline$\$ 63,000$ & 17.19 & 19.95 & \\
\hline Hospital region & & & $<0.001$ \\
\hline Northwest & 18.87 & 18.54 & \\
\hline Midwest & 22.86 & 20.03 & \\
\hline South & 37.92 & 38.93 & \\
\hline West & 20.35 & 22.5 & \\
\hline Hospital location & & & $<0.001$ \\
\hline Rural & 9.65 & 8.44 & \\
\hline Urban & 90.35 & 91.56 & \\
\hline Hospital size & & & $<0.001$ \\
\hline Small & 24.65 & 20.64 & \\
\hline Medium & 31.41 & 31.08 & \\
\hline Large & 43.94 & 48.29 & \\
\hline Teaching hospital & 61.94 & 64.81 & $<0.001$ \\
\hline Race & & & $<0.001$ \\
\hline White & 61.68 & 64.37 & \\
\hline Black & 23.42 & 10.38 & \\
\hline Hispanic & 9.87 & 18.00 & \\
\hline Asian or Pacific Islander & 1.24 & 2.93 & \\
\hline Native American & 1.21 & 0.85 & \\
\hline Other & 2.59 & 3.46 & \\
\hline \multicolumn{4}{|l|}{ Comorbidities } \\
\hline $\mathrm{CHF}$ & 2.75 & 7.76 & $<0.001$ \\
\hline Cirrhosis & 5.55 & 2.5 & $<0.001$ \\
\hline
\end{tabular}


Table 2 (Continued)

\begin{tabular}{lccc}
\hline \multirow{2}{*}{ Variable } & \multicolumn{3}{c}{$\mathrm{n}(\%)$} \\
\cline { 2 - 4 } & AAP $140,195(25.3 \%)$ & ABP $96,540(17.44 \%)$ & P-value \\
\hline Type 1 diabetes & 0.69 & 0.33 & $<0.001$ \\
Type 2 diabetes & 13.11 & 22.37 & $<0.001$ \\
CKD & 3.25 & 10.19 & $<0.001$ \\
ESRD & 0.34 & 1.61 & $<0.001$ \\
Obese & 8.24 & 26.26 & $<0.001$ \\
Smoker & 66.17 & 30.75 & $<0.001$ \\
\hline CHF congestive heart failure: CKD, chronic kidney disease. ESRD, end-stage renal disease & & \\
\hline
\end{tabular}

\section{Hospitalization charges and costs}

The mean hospitalization charges and cost for patients admitted with AP were $\$ 41,018$ and $\$ 10,153$, respectively. Patients with ABP had significantly higher adjusted mean total hospitalization charges and costs $(\$ 61,182$ and $\$ 14,941)$ compared to patients with AAP $(\$ 37,982$ and $\$ 9,583)$. The mean adjusted difference in hospitalization charges was $\$+19,958(\mathrm{P}<0.001)$, and the mean adjusted difference in hospitalization costs was $\$+4,848(\mathrm{P}<0.001)$.

\section{Shock}

Among all patients with AP, $0.83 \%$ developed shock during their hospital stay. The proportion of patients developing shock was $0.96 \%$ in AAP and $1.06 \%$ in ABP. Although the difference was small, patients with $\mathrm{ABP}$ had a significantly lower likelihood of shock compared to patients with AAP (aOR 0.75, 95\%CI 0.59-0.95; $\mathrm{P}=0.02$ ).

\section{Sepsis}

Among all patients with AP, 3.2\% developed sepsis during their hospital stay. The proportion of patients developing sepsis was $3.97 \%$ in AAP and 3.35\% in ABP. There was no significant difference in the likelihood of sepsis between these groups (aOR 0.90, 95\%CI 0.80-1.01; $\mathrm{P}=0.10$ ).

\section{AKI}

Among all patients with AP, 10.98\% developed AKI during their hospital stay. The proportion of patients developing AKI was $10.34 \%$ in AAP and $11.54 \%$ in ABP. Patients with ABP were significantly less likely to have AKI compared to patients with AAP (aOR 0.76, 95\%CI 0.71$0.82 ; \mathrm{P}<0.001)$.

\section{ICU admission}

Among all patients with AP, $1.35 \%$ patients needed ICU admission during their hospital stay. The proportion of patients needing an ICU stay was $1.96 \%$ in AAP and $1.71 \%$ in ABP. Patients with ABP were significantly less likely to be admitted to the ICU compared to patients with AAP (aOR 0.74, 95\%CI 0.62-0.88; $\mathrm{P}=0.001)$.

\section{Pancreatic drainage}

Overall, $0.60 \%$ of patients with AP underwent pancreatic drainage; $0.42 \%$ of patients with AAP underwent pancreatic drainage as compared to $0.45 \%$ of patients with ABP. On multivariate analysis, there was no significant difference in the likelihood of pancreatic drainage in these groups (aOR 1.15, 95\%CI 0.81-1.62; $\mathrm{P}=0.42$ ).

\section{Home discharge}

A total of $466,744(87.49 \%)$ patients with AP were discharged home after the hospitalization; $86.12 \%$ of patients with AAP and $83.39 \%$ of patients with ABP were discharged home. On multivariate analysis, patients with ABP were more likely to be discharged home than patients with AAP (aOR 1.26, 95\%CI 1.18-1.34; $\mathrm{P}<0.001)$.

\section{Discussion}

Our retrospective analysis of the NIS database showed that there was no significant difference in all-cause inhospital mortality between patients with AAP and ABP. Patients with ABP had a longer LOS, higher hospitalization charges/costs, and a significantly smaller likelihood of shock, AKI, and ICU admission. However, it is pertinent to remember that, while statistically significant, the 
Table 3 Outcomes in patients with acute pancreatitis: acute biliary pancreatitis (ABP) versus acute alcohol-induced pancreatitis (AAP)

\begin{tabular}{|c|c|c|c|c|}
\hline & \multirow[t]{2}{*}{ Outcomes } & \multicolumn{3}{|c|}{ Study population with acute pancreatitis $n=553,480$} \\
\hline & & AAP 140,195 & ABP 96,540 & P-value \\
\hline \multirow[t]{3}{*}{1.} & In-hospital mortality & $0.42 \%$ & $0.82 \%$ & \\
\hline & $\mathrm{uOR}$ & Ref & $1.96(1.54-2.49)$ & $<0.001$ \\
\hline & $\mathrm{aOR}$ & Ref & $0.95(0.69-1.31)$ & 0.79 \\
\hline \multirow[t]{2}{*}{2.} & Mean length of stay (LOS-days) & 4.38 & 5.10 & \\
\hline & Adjusted coefficient & Ref & $0.48(0.35-0.60)$ & $<0.001$ \\
\hline \multirow[t]{2}{*}{3.} & Mean total charge & $\$ 37,982$ & $\$ 61,182$ & \\
\hline & Adjusted coefficient & Ref & $\$ 19,958$ & $<0.001$ \\
\hline \multirow[t]{2}{*}{4.} & Mean total cost & $\$ 9,583$ & $\$ 14,941$ & \\
\hline & Adjusted coefficient & Ref & $\$ 4,848$ & $<0.001$ \\
\hline \multirow[t]{3}{*}{5.} & Shock & $0.95 \%$ & $1.05 \%$ & \\
\hline & $\mathrm{uOR}$ & Ref & $1.10(0.92-1.32)$ & 0.28 \\
\hline & $\mathrm{aOR}$ & Ref & $0.75(0.59-0.95)$ & 0.02 \\
\hline \multirow[t]{3}{*}{6.} & Sepsis & $3.96 \%$ & $3.34 \%$ & \\
\hline & $\mathrm{uOR}$ & Ref & $0.83(0.75-0.92)$ & $<0.001$ \\
\hline & $\mathrm{aOR}$ & Ref & $0.90(0.80-1.01)$ & 0.10 \\
\hline \multirow[t]{3}{*}{7.} & ICU Admission & $1.96 \%$ & $1.70 \%$ & \\
\hline & $\mathrm{uOR}$ & Ref & $0.86(0.75-0.99)$ & 0.045 \\
\hline & $\mathrm{aOR}$ & Ref & $0.74(0.62-0.88)$ & 0.001 \\
\hline \multirow[t]{3}{*}{8.} & AKI & $10.33 \%$ & $11.53 \%$ & \\
\hline & $\mathrm{uOR}$ & Ref & $1.13(1.06-1.20)$ & $<0.001$ \\
\hline & $\mathrm{aOR}$ & Ref & $0.76(0.71-0.82)$ & $<0.001$ \\
\hline \multirow[t]{3}{*}{9.} & Pancreatic drainage & $0.42 \%$ & $0.45 \%$ & \\
\hline & $\mathrm{uOR}$ & Ref & $1.07(0.81-1.42)$ & 0.61 \\
\hline & $\mathrm{aOR}$ & Ref & $1.15(0.81-1.62)$ & 0.42 \\
\hline \multirow[t]{3}{*}{10.} & Home discharge & $85.68 \%$ & $82.62 \%$ & \\
\hline & $\mathrm{uOR}$ & Ref & $0.80(0.76-0.85)$ & $<0.001$ \\
\hline & $\mathrm{aOR}$ & Ref & $1.26(1.18-1.34)$ & $<0.001$ \\
\hline \multirow[t]{3}{*}{11.} & Pancreatic necrosis & $2.62 \%$ & $1.97 \%$ & \\
\hline & $\mathrm{uOR}$ & Ref & $0.74(0.65-0.85)$ & $<0.001$ \\
\hline & $\mathrm{aOR}$ & Ref & $0.89(0.76-1.05)$ & 0.19 \\
\hline
\end{tabular}

$\overline{I C U}$, intensive care unit; AKI, acute kidney injury; Ref, reference; $u O R$, unadjusted odds ratio; aOR, adjusted odds ratio; LOS, length of stay

differences in some of these outcomes were quite modest and indicate a somewhat similar clinical course between the 2 groups. There have been previous studies comparing the outcomes of alcohol-induced and gallstone pancreatitis in patients admitted to tertiary centers, but the sample size was in the hundreds [20,21]. In comparison, we were able to look at 553,480 adult patients with AP across the nation, including 140,195 (25.3\%) with AAP and 96,540 (17.44\%) with ABP.

Previous studies have shown a correlation between sex and the etiology of AP, with a higher percentage of male patients having AAP and a higher percentage of females having ABP $[8,22]$. Furthermore, patients with AAP were found to be younger than those with ABP [8]. Our study shows similar results: patients with $\mathrm{ABP}$ were more likely to be female $(59.4 \%$ vs. $30.73 \%, \mathrm{P}<0.001)$ and older $(57.03$ vs. 45.31 years, $\mathrm{P}<0.001)$ compared to patients with AAP. This is consistent with the current evidence that biliary stone disease is almost twice as likely in females than in males, and that age has a positive correlation with the frequency of the disease [23]. In addition, patients with ABP were found to have a significantly higher prevalence of CHF, Type 2 
DM, CKD, ESRD, and obesity, while patients with AAP had significantly more liver cirrhosis and were more likely to be smokers.

In our study, there was no significant difference in all-cause in-hospital mortality between patients with AAP and ABP (aOR 0.95, 95\%CI 0.69-1.31; P=0.79). Multiple studies have reported a similar absence of mortality difference between the 2 groups $[8,13,20]$. However, some studies reported a more severe course and higher mortality in the alcohol group [11,12], while other, older studies reported a more severe outcome in the biliary group $[9,10]$.

We found that a higher percentage of patients with AAP had pancreatic necrosis compared to ABP (2.62\% vs. $1.97 \%, \mathrm{P}<0.001$ ), but the difference was not significant on multivariate analysis. There was no significant difference in infected necrosis between the 2 groups. Samanta et al also reported a similarly higher incidence of necrosis in the alcohol group in their study $(90.2 \%$ vs. $84.1 \%, \mathrm{P}=0.05)$; however, their reporting of almost $90 \%$ pancreatic necrosis is extremely high compared to the estimate of $4-47 \%$ in the general population [24]. The smaller number observed in our study is probably due to the discrepancy in billing codes submitted by the physicians. It is possible that complications such as necrosis and infection are submitted as secondary diagnoses, and may have been missed by our inclusion of primary diagnosis only [20]. We also demonstrated that patients with ABP had a lower likelihood of shock, AKI, or ICU admission, and they were also more likely to be discharged home. Samanta et al reported that the development of organ failure, shock, and AKI was similar between the alcohol and the biliary group [20], while Cho et al reported more severe disease in the alcohol group [21]. Patients with AAP are more likely to have a history of prolonged and heavy drinking, so pancreatic damage is likely to be already present [25], making these patients more susceptible to ductal disruption with leakage of pancreatic juice, leading to further insults. On the other hand, patients with ABP are more likely to present with a more acute episode of biliary duct obstruction with a previously relatively normal pancreas, and procedures like endoscopic retrograde cholangiopancreatography (ERCP) and cholecystectomy can be curative.

Patients with ABP also had a longer LOS and higher hospitalization charges and costs compared to the patients with AAP. This is not surprising, as patients with ABP are more likely to undergo imaging studies, including magnetic resonance cholangiopancreatography, and endoscopic procedures, such as ERCP, that can add to the overall LOS and costs. However, the difference in LOS between the 2 groups was less than $12 \mathrm{~h}$ (0.48 days), and its clinical relevance is debatable. A recent database study of patients with ABP showed that the hospitalization cost was increasing, even though use of ERCP in ABP patients was decreasing [26].

Our study has multiple limitations. Our data were obtained using NIS, an administrative, claim-based database. It has been previously documented that missing or inaccurately entered codes are part of claim-based databases such as NIS.
We only included patients with a primary diagnosis (DX1) of acute pancreatitis to increase the specificity of our study. In doing so, some of the other pertinent clinical variables coded as secondary diagnoses (DX2-40) may have been missed. As shown by Xiao et al, the positive predictive value of ICD codes (ICD 9 and 10) was 0.78 for incident episodes of AP [27]. Our study was performed using ICD-10 codes only, and we believe this enabled us to arrive at a more accurate estimate. In our study, $42.74 \%$ of patients had pancreatitis due to alcohol or biliary disease, less than what has generally been reported in previous studies [6-8]. More than half of the cases in our study (51.83\%) were classified as "other/unspecified acute pancreatitis", which may have resulted in this discrepancy. There is a chance that discharges due to AAP or ABP were misclassified into this category, leading to skewing of the results and limiting the accuracy of our findings. This nondifferential misclassification bias may have prevented us from finding a closer estimate of the outcomes (OR). Furthermore, there was no randomization between the 2 groups in our study, as the data were obtained retrospectively from NIS. NIS still stratifies patients according to race (White, Black, Hispanic, etc.), probably an outdated classification, and the preferred stratification would have been according to ethnicity (Hispanic/Latino vs. non-Hispanic/Latino) per NIH racial/ ethnic category guidelines. We provide our data per available NIS classification.

As discussed above, there is significant heterogeneity in the evidence regarding a difference in outcomes of AP based on etiology [21,28-31]. Furthermore, there is a paucity of data on the outcome differences between the most common types: AAP and ABP. The major strength of our study is that it compares the outcomes of 553,480 patients with AP across the nation, while focusing on in-hospital outcomes of alcohol-induced and biliary acute pancreatitis. This large sample size minimizes the likelihood of a $\beta$ error. The results of our study are also representative of real-life practice in US hospitals, and can thus be generalized to the US population. Hence, our study helps to bridge the gap in this knowledge.

In conclusion, our study showed no difference in mortality when comparing patients with AAP and ABP. When looking at resource utilization, patients with ABP had a longer LOS and higher hospitalization charges and costs compared to patients with AAP. Patients with ABP were less likely to develop shock, AKI, or be admitted to the ICU when compared to patients with AAP and they were also more likely to be discharged home. While statistically significant, the differences between several of these outcomes were small and the limitations of this study should be kept in mind when interpreting the results. Nevertheless, clinicians should be wary of these potential adverse outcomes in patients with AAP, and care should be focused on close hemodynamic and electrolyte monitoring for the earlier detection and prevention of these comorbidities. Further prospective studies and meta-analysis will help successfully guide clinical practice in the future. 


\section{Summary Box}

\section{What is already known:}

- Acute pancreatitis (AP) is the most common cause of gastroenterological admission, with alcoholinduced and biliary pathology being the most common etiologies

- Acute alcohol-induced pancreatitis (AAP) and acute biliary pancreatitis $(\mathrm{ABP})$ are 2 distinct entities with different causes, pathogenesis, and clinical course

- There is still significant heterogeneity regarding differences in the outcomes of AP based on etiology, primarily AAP vs. ABP

\section{What the new findings are:}

- In the National Inpatient Sample discharge database based on ICD-10 coding, AAP and ABP make up less than $50 \%$ of all causes of AP

- There is no difference in in-hospital mortality rates between AAP and ABP

- Compared to AAP, patients with ABP do better in terms of other in-hospital outcomes such as shock, acute kidney injury and intensive care unit admission, and incur lower hospitalization costs, to a small but statistically significant degree

\section{References}

1. Lee PJ, Papachristou GI. New insights into acute pancreatitis. Nat Rev Gastroenterol Hepatol 2019;16:479-496.

2. Petrov MS, Yadav D. Global epidemiology and holistic prevention of pancreatitis. Nat Rev Gastroenterol Hepatol 2019;16:175-184.

3. Yadav D, Lowenfels AB. The epidemiology of pancreatitis and pancreatic cancer. Gastroenterology 2013;144:1252-1261.

4. Peery AF, Crockett SD, Murphy CC, et al. Burden and cost of gastrointestinal, liver, and pancreatic diseases in the United States: update 2018. Gastroenterology 2019;156:254-272.

5. Wadhwa V, Patwardhan S, Garg SK, Jobanputra Y, Lopez R, Sanaka MR. Health care utilization and costs associated with acute pancreatitis. Pancreas 2017;46:410-415.

6. Manrai M, Kochhar R, Gupta V, et al. Outcome of acute pancreatic and peripancreatic collections occurring in patients with acute pancreatitis. Ann Surg 2018;267:357-363.

7. Tenner S, Baillie J, DeWitt J, Vege SS; American College of Gastroenterology. American College of Gastroenterology guideline: management of acute pancreatitis. Am J Gastroenterol 2013;108:1400-1415; 1416.

8. Gullo L, Migliori M, Oláh A, et al. Acute pancreatitis in five European countries: etiology and mortality. Pancreas 2002;24:223-227.

9. Ranson JH, Rifkind KM, Roses DF, Fink SD, Eng K, Spencer FC. Prognostic signs and the role of operative management in acute pancreatitis. Surg Gynecol Obstet 1974;139:69-81.

10. Frey CF. Gallstone pancreatitis. Surg Clin North Am 1981;61:923-938.
11. Kim DB, Chung WC, Lee JM, Lee KM, Oh JH, Jeon EJ. Analysis of factors associated with the severity of acute pancreatitis according to etiology. Gastroenterol Res Pract 2017;2017:1219464.

12. Zhu Y, Pan X, Zeng H, et al. A study on the etiology, severity, and mortality of 3260 patients with acute pancreatitis according to the revised Atlanta classification in Jiangxi, China over an 8-year period. Pancreas 2017;46:504-509.

13. Uhl W, Isenmann R, Curti G, Vogel R, Beger HG, Büchler MW. Influence of etiology on the course and outcome of acute pancreatitis. Pancreas 1996;13:335-343.

14. Available from: https://www.hcup-us.ahrq.gov/db/nation/nis/ NIS_Introduction_2017.jsp [Accessed 30 October 2020].

15. CDC ICD-10. Available from: https://www.cdc.gov/nchs/icd/ icd10cm_pcs_background.htm [Accessed 30 October 2020].

16. Bilal M, Tayyem O, Saraireh H, Chowdhry M, Guturu P, Abougergi MS. Upper gastrointestinal hemorrhage is associated with poor outcomes among patients with acute cholangitis: a nationwide analysis. Eur J Gastroenterol Hepatol 2019;31:586-592.

17. Arora V, Moriates C, Shah N. The challenge of understanding health care costs and charges. AMA J Ethics 2015;17:1046-1052.

18. US Agency for Healthcare Research and Quality. Cost-to-charge ratio files. Available from: https://www.hcup-us.ahrq.gov/db/state/ costtocharge.jsp [Accessed 30 October 2020].

19. Abougergi MS, Peluso H, Saltzman JR. Thirty-day readmission among patients with non-variceal upper gastrointestinal hemorrhage and effects on outcomes. Gastroenterology 2018;155:38-46.

20. Samanta J, Dhaka N, Gupta P, et al. Comparative study of the outcome between alcohol and gallstone pancreatitis in a highvolume tertiary care center. JGH Open 2019;3:338-343.

21. Cho JH, Kim TN, Kim SB. Comparison of clinical course and outcome of acute pancreatitis according to the two main etiologies: alcohol and gallstone. BMC Gastroenterol 2015;15:87.

22. Imrie CW. Observations on acute pancreatitis. $\mathrm{Br} J$ Surg 1974;61:539-544.

23. Stinton LM, Shaffer EA. Epidemiology of gallbladder disease: cholelithiasis and cancer. Gut Liver 2012;6:172-187.

24. Banks PA, Freeman ML; Practice Parameters Committee of the American College of Gastroenterology. Practice guidelines in acute pancreatitis. Am J Gastroenterol 2006;101:2379-2400.

25. Singh M, Simsek H. Ethanol and the pancreas. Current status. Gastroenterology 1990;98:1051-1062.

26. Bilal M, Kline KT, Trieu JA, et al. Trends in sameadmission cholecystectomy and endoscopic retrograde cholangiopancreatography for acute gallstone pancreatitis: a nationwide analysis across a decade. Pancreatology 2019;19:524-530.

27. Xiao AY, Tan ML, Plana MN, Yadav D, Zamora J, Petrov MS. The use of international classification of diseases codes to identify patients with pancreatitis: a systematic review and meta-analysis of diagnostic accuracy studies. Clin Transl Gastroenterol 2018;9:191.

28. Berger Z, Mancilla C, Tobar E, et al. Acute pancreatitis in Chile: A multicenter study on epidemiology, etiology and clinical outcome. Retrospective analysis of clinical files. Pancreatology 2020;20:637-643.

29. Kamal A, Akshintala VS, Kamal MM, et al. Does etiology of pancreatitis matter? differences in outcomes among patients with post-endoscopic retrograde cholangiopancreatography, acute biliary, and alcoholic pancreatitis. Pancreas 2019;48:574-578.

30. Weitz G, Woitalla J, Wellhöner P, Schmidt K, Büning J, Fellermann K. Does etiology of acute pancreatitis matter? A review of 391 consecutive episodes. JOP 2015;16:171-175.

31. Roberts SE, Williams JG, Meddings D, Goldacre MJ. Incidence and case fatality for acute pancreatitis in England: geographical variation, social deprivation, alcohol consumption and aetiology-a record linkage study. Aliment Pharmacol Ther 2008;28:931-941. 


\section{Supplementary material}

Supplementary Table 1 The International Classification of Diseases, $10^{\text {th }}$ revision, (ICD-10) diagnostic and procedural codes used to generate search results

\begin{tabular}{ll}
\hline Acute pancreatitis & K85.XX \\
\hline $\begin{array}{l}\text { Acute biliary pancreatitis } \\
\text { Acute alcoholic pancreatitis }\end{array}$ & K851, K8510, K8511,K8512 \\
\hline Shock & R65.21; R57.1; R57.8; R57.9 \\
\hline Acute kidney injury & N17.0; N17.1; N17.2; N17.8; N17.9 \\
\hline Mechanical ventilation (A) & 5A1935Z; 5A1945Z; 5A1955Z \\
\hline Vasopressor use (B) & 3E030XZ; 3E033XZ; 3E040XZ; \\
& 3E043XZ; 3E050XZ; 3E053XZ; \\
& 3E060XZ; 3E063XZ \\
\hline Pancreatic drainage & 0F9G00Z; 0F9G0ZX; 0F9G0ZZ; \\
& 0F9G30Z; 0F9G3ZX; 0F9G3ZZ; \\
& 0F9G40Z; 0F9G4ZX; 0F9G4ZZ; \\
& 0F9G80Z; 0F9G8ZX; 0F9G8ZZ; \\
\hline
\end{tabular}

$A+B$ : intensive care unit admission 